\title{
Melioidosis in Brunei Darussalam
}

\author{
Ketan Pande 1,*(D), Khairul Azmi Abd Kadir ${ }^{1}$, Rosmonaliza Asli ${ }^{2}$ and Vui Heng Chong ${ }^{2}$ \\ 1 Department of Orthopaedics, Raja Isteri Pengiran Anak Saleha Hospital, Bandar Seri Begawan BG 1710, \\ Negara Brunei Darussalam; khairulazmiabdkadir@gmail.com \\ 2 Department of Medicine, Raja Isteri Pengiran Anak Saleha Hospital, Bandar Seri Begawan BG 1710, \\ Negara Brunei Darussalam; ROSMONALIZA.ASLI@moh.gov.bn (R.A.); \\ vuiheng.chong@moh.gov.bn (V.H.C.) \\ * Correspondence: ketanpande@yahoo.com; Tel.: +673-8610747
}

Received: 30 January 2018; Accepted: 14 February 2018; Published: 19 February 2018

\begin{abstract}
Melioidosis continues to be a major health care problem in Brunei Darussalam. The age of patients, gender distribution, risk factors, and clinical presentations are similar to those reported from other countries in the region. The incidence of melioidosis was high during the wet months and in the Temburong district, which has the highest annual rainfall. In spite of adequate facilities for diagnosis and treatment, the mortality remains high (27\%). Women and those presenting with septic shock had higher mortality. There is a case for making melioidosis a notifiable disease in Brunei Darussalam. Coordinated efforts between policy-makers and various stakeholders are required to effectively combat the disease.
\end{abstract}

Keywords: melioidosis; Burkholderia pseudomallei; epidemiology; diagnosis; treatment

\section{Introduction}

Melioidosis is endemic in many countries of Southeast Asia, including Brunei Darussalam and in northern Australia. It is estimated that $40 \%$ of all cases occur in the East Asia Pacific region [1].

Brunei Darussalam is a country in Southeast Asia, located in the northeastern part of Borneo island, with geographical coordinates of $4^{\circ} 30^{\prime} \mathrm{N} 114^{\circ} 40^{\prime} \mathrm{E}$. The country is divided into four districts: Brunei Muara, Tutong, Kuala Belait, and Temburong. The climate in Brunei Darussalam is tropical equatorial and humid subtropical at higher altitudes with heavy rainfall [2]. The annual average rainfall is more than $2300 \mathrm{~mm}$ for the whole of Brunei Darussalam and increases inland, with altitude, to more than $4000 \mathrm{~mm}$ with maximum rainfall in the Temburong district [3]. The mean monthly rainfall follows seasonal patterns with two maxima and two minima. The first maximum is from October to January, with December being the wettest month, while the second minor maximum is from May to July, with May being relatively wetter [4].

In line with the aims of this special issue on melioidosis, this article addresses various aspects of the disease in Brunei Darussalam. It is divided into the following parts: (i) a review of available literature on melioidosis from Brunei Darussalam; (ii) the current approaches to diagnosis, management, and prevention; (iii) epidemiological study of melioidosis with data obtained from 2015-2016; (iv) melioidosis in animals; and (v) future challenges.

\section{Review of Literature from Brunei Darussalam}

The first published report on melioidosis (24 cases) in Brunei Darussalam was by Luqman et al. [5]. It was more prevalent in the agricultural districts and in the period $(1993,1994)$ the rate increased from 2.9 to $5.6 / 100,000$. The majority $(79.2 \%)$ had risk factors, mostly diabetes mellitus. Septicaemia was the presenting feature in $75 \%$ of cases, with $62.6 \%$ having multi-organ involvement. The mortality rate was $20.8 \%$ and on follow-up, $60 \%$ of patients presented with relapse of the disease. 
The many and varied radiological manifestations of melioidosis in Brunei Darussalam were reviewed by Lim and Chong [6]. Multiple organ involvement, especially the lungs, liver, and spleen, were common. The 'honeycomb' appearance of abscesses, especially large abscesses and those seen in the liver, was characteristic of melioidosis.

Chong et al. reported the characteristics of pancreatic melioidosis $(4 / 65$ cases who had undergone CT imaging, median age 29.5 years (range 25-48 years)). Pancreatic involvement ranged from multi-focal micro-abscesses to focal large abscesses, and associated findings included splenic vein thrombosis, peri-pancreatic inflammation, and peri-pancreatic fat streaking. Pancreatic involvement was typically part of multi-organ involvement [7].

Pande and $\mathrm{Hj}$ Abdul Kadir reported their experience with melioidosis affecting the extremities $(n=14 / 48$ (29.1\%); median age 45 years (range $14-55)$. The majority $(n=13,92.8 \%)$ were men, with $35.7 \%(n=5)$ being expatriates working as labourers. Septic arthritis was the most common presentation ( $n=5)$, followed by cellulitis, abscess, and osteomyelitis ( $n=3$ each) [8].

The antibiograms of Burkholderia pseudomallei from Brunei Darussalam over a period of ten years (679 isolates from 623 patients) showed that carbapenems, third-generation cephalosporins (ceftazidime), piperacillin, and chloramphenicol had the highest susceptibility rates of between $98 \%$ and $100 \%$. Amoxicillin-clavulanic acid had a moderate susceptibility rate (76-100\%) and quinolones (ciprofloxacin, 32-68\%) and co-trimoxazole (6-54\%) had the lowest susceptibility rates. It is, however, recognized that the disk diffusion method overestimates the resistance rate for co-trimoxazole [9]. The gentamicin susceptibility of the isolates was not reported.

A case of right frontal lobe brain abscess due to co-infection with B. pseudomallei and Cryptococcus neoformans in a patient with underlying systemic lupus erythematosus was reported by Samad et al. [10]. The patient was non-diabetic but was on steroid therapy and the diagnosis was confirmed only after surgical drainage.

Chong et al. reported their experience with urogenital melioidosis $(n=13$, nine new cases, four relapses; median age 38 (range 29-63 years)). The kidneys were involved in $72.3 \%$ followed by the prostate $(60 \%)$. Testicular and seminal vesicle involvement was noted in one case each. There was no involvement of the gynaecological system [11].

In the clinical case series mentioned above, a male preponderance was noted, with diabetes mellitus being the most common risk factor $[7,8,11]$.

\section{Current Approach to Diagnosis, Management, and Prevention}

\subsection{Microbiological Diagnosis}

In Brunei Darussalam, the diagnosis of melioidosis is confirmed by the isolation of B. pseudomallei from clinical specimens, such as blood, sputum, abscess aspirates, pericardial fluid, skin lesions, or other clinical specimens. The clinical specimens are collected during the initial admission to the hospital. Positive blood culture bottles are subcultured onto blood agar and MacConkey agar; urine specimens are inoculated onto cystine lactose electrolyte-deficient (CLED) agar; respiratory specimens, onto blood agar, MacConkey agar, and chocolate agar; and pus specimens into cooked meat broth. These are incubated at $37^{\circ} \mathrm{C}$ for at least $24 \mathrm{~h}$ before being examined. For pus specimens, subsequent subcultures are made from the broth onto blood agar and MacConkey agar and reincubated at $37^{\circ} \mathrm{C}$ for at least another $24 \mathrm{~h}$ before re-examination. Selective agars, such as those studied by Peacock et al. [12], are not used in Brunei Darussalam, which means that in some cases growth of B. pseudomallei may be missed. In a patient with high clinical suspicion of melioidosis, but with negative cultures, reactivity in a B. pseudomallei serological test (sent abroad) is taken as a strong evidence of the disease.

The cultures are then examined for colony morphology and Gram-staining. Morphologically, B. pseudomallei is identified as large, wrinkled colonies that have a metallic appearance with an earthy odour. On Gram staining, the organism appears as Gram-negative bacilli with bipolar staining, which gives a characteristic 'safety pin' appearance. The cultured colonies are further identified using the 
VITEK system, a commercial bacterial identification system. However, it is noted that with the use of the VITEK system there is potential for misidentification of B. pseudomallei as Burkholderia cepacia, which is regionally dependent [13]. This is of particular significance as the study reporting this was from Malaysian Borneo, on the same island as Brunei Darussalam.

All of the isolated organisms are tested for antibiotic susceptibility using Etest and, at present, the results of susceptibility testing are not validated by an external reference laboratory.

\subsection{Management}

Patients who are clinically suspected to have melioidosis, particularly those with a background history of uncontrolled diabetes mellitus, are empirically treated with an antibiotic that has activity against B. pseudomallei, i.e., either with intravenous (IV) ceftazidime (up to 2 g every 8 h) or, for clinically-severe cases, with IV imipenem (up to $1 \mathrm{~g}$ every $8 \mathrm{~h}$ ) or IV meropenem (up to $1 \mathrm{~g}$ every $8 \mathrm{~h}$ ) while waiting for culture results to become available.

In patients with confirmed B. pseudomallei bacteraemia, and in those patients who were empirically treated with either IV imipenem or meropenem and who have been stable for $48 \mathrm{~h}$, the IV antibiotic is de-escalated to IV ceftazidime. Further investigations, like chest radiographs, ultrasound of the abdomen and pelvis, or computed tomography (CT) scan of the thorax, abdomen, and pelvis (CT-TAP), are usually performed to exclude deep-seated infection with organ abscesses that may be drainable.

The antibiotic treatment for melioidosis comprises two phases, i.e., the intensive phase and the eradication phase. In the intensive phase, IV antimicrobial therapy with either IV ceftazidime or IV meropenem or imipenem is recommended for at least 10-14 days for pulmonary disease or if there are no other obvious deep-seated sources. The intensive phase may be extended to at least $4-6$ weeks with drainage of deep-seated abscesses. The patient is then continued on to the eradication phase with oral co-trimoxazole $(5 \mathrm{mg} / \mathrm{kg}$ of the trimethoprim component every $12 \mathrm{~h}$ ) as the first line antimicrobial therapy for at least 12 weeks in total. If co-trimoxazole is contraindicated or the patient develops adverse reactions to the medication, then the second-line treatment would be with oral co-amoxiclav (625 mg every $8 \mathrm{~h}$ ) and oral doxycycline ( $2 \mathrm{mg} / \mathrm{kg}$ every $12 \mathrm{~h}$ ) to complete a total minimum of 12 weeks. However, clinicians need to be aware of the risk of treatment failure with this second-line eradication phase regimen $[14,15]$. Currently no post-exposure prophylaxis is offered in Brunei Darussalam.

\subsection{Preventive Measures}

Patients who are admitted with suspected or confirmed pulmonary melioidosis are nursed in droplet isolation for at least $24 \mathrm{~h}$ after appropriate antibiotics are commenced.

In the laboratory setting, biosafety level 3 practices are adopted while handling and processing specimens [16].

\section{Epidemiological Study of Melioidosis with Data Obtained from 2015-2016}

\subsection{Material and Methods}

All culture-positive cases of melioidosis recorded between January 2015 and December 2016 were retrieved from the electronic database of the Clinical Microbiology Laboratory, Department of Laboratory Services, RIPAS Hospital. All patients were investigated and managed as per the protocol mentioned in the previous section.

Demographic, clinical and diagnostic information (laboratory and imaging) was collected through the Hospital Information Management System (BruHIMS). The study was approved by the Medical and Health Research and Ethics Committee (MHREC).

Data are presented as descriptive statistics. Wherever possible, differences between groups were analysed using the chi-square test. A $p$-value of $<0.05$ was considered significant. 


\subsection{Results}

\subsubsection{Demographics}

A total of 115 cases were detected in $2015(n=46)$ and $2016(n=69)$. There were $84(73 \%)$ male and $31(27 \%)$ female patients with an age range from 2 to 86 years (median 48 years).

There were four (3.5\%) patients under the age of 15 years, all with no risk factors, who presented with abscesses in soft tissue $(n=3)$ and neck $(n=1)$. They were admitted in the months of January, April, August, and December.

Excluding a two-year-old expatriate patient, the median age of expatriate patients was 41.5 years $(n=25)$. The age range for Bruneian patients $(n=89)$ was 14 to 86 years with a median age of 52 years.

The ethnic distribution of the cases is given in Table 1.

Table 1. Ethnic distribution of cases of melioidosis.

\begin{tabular}{ccc}
\hline Country & No. & $\mathbf{\%}$ \\
\hline Malay & 88 & 76.5 \\
Chinese: Bruneian & 1 & 0.9 \\
Expatriates & 26 & 22.6 \\
Bangladesh & 10 & 8.7 \\
Indonesia & 8 & 7 \\
India & 4 & 3.5 \\
Philippines & 1 & 0.9 \\
Thailand & 1 & 0.9 \\
Nepal & 1 & 0.9 \\
Malaysia & 1 & 0.9 \\
\hline
\end{tabular}

During 2015-2016, the ethnic distribution of the population of Brunei Darussalam was Malay $(65.7 \%)$, Chinese (10.3\%), and expatriates (23.9\%) [17].

The overall incidence rates per 100,000 population in 2015 and 2016 were 11 and 16.3, respectively.

The number of cases and incidence rate per 100,000 population from each of the four districts is presented in Table 2.

Table 2. District-wise incidence of melioidosis/100,000 population for 2015-2016.

\begin{tabular}{ccccccc}
\hline District & Pop. 2015 & Cases & Inc/100,000 & Pop. 2016 & Cases & Inc/100,000 \\
\hline Muara & 288,400 & 33 & 11.44 & 292,705 & 51 & 17.42 \\
Tutong & 48,700 & 9 & 18.48 & 49,438 & 8 & 16.18 \\
Kuala Belait & 69,000 & 2 & 2.89 & 69,992 & 6 & 8.57 \\
Temburong & 10,400 & 2 & 19.23 & 10,543 & 4 & 37.93 \\
\hline
\end{tabular}

Population figures obtained from http:/ / www.depd.gov.bn/sitePages/Population.aspx [17].

The incidence was highest in the Temburong district in both years (Figure 1). Except in the Tutong district, there was an increase in incidence for the remaining districts from 2015 to 2016. 


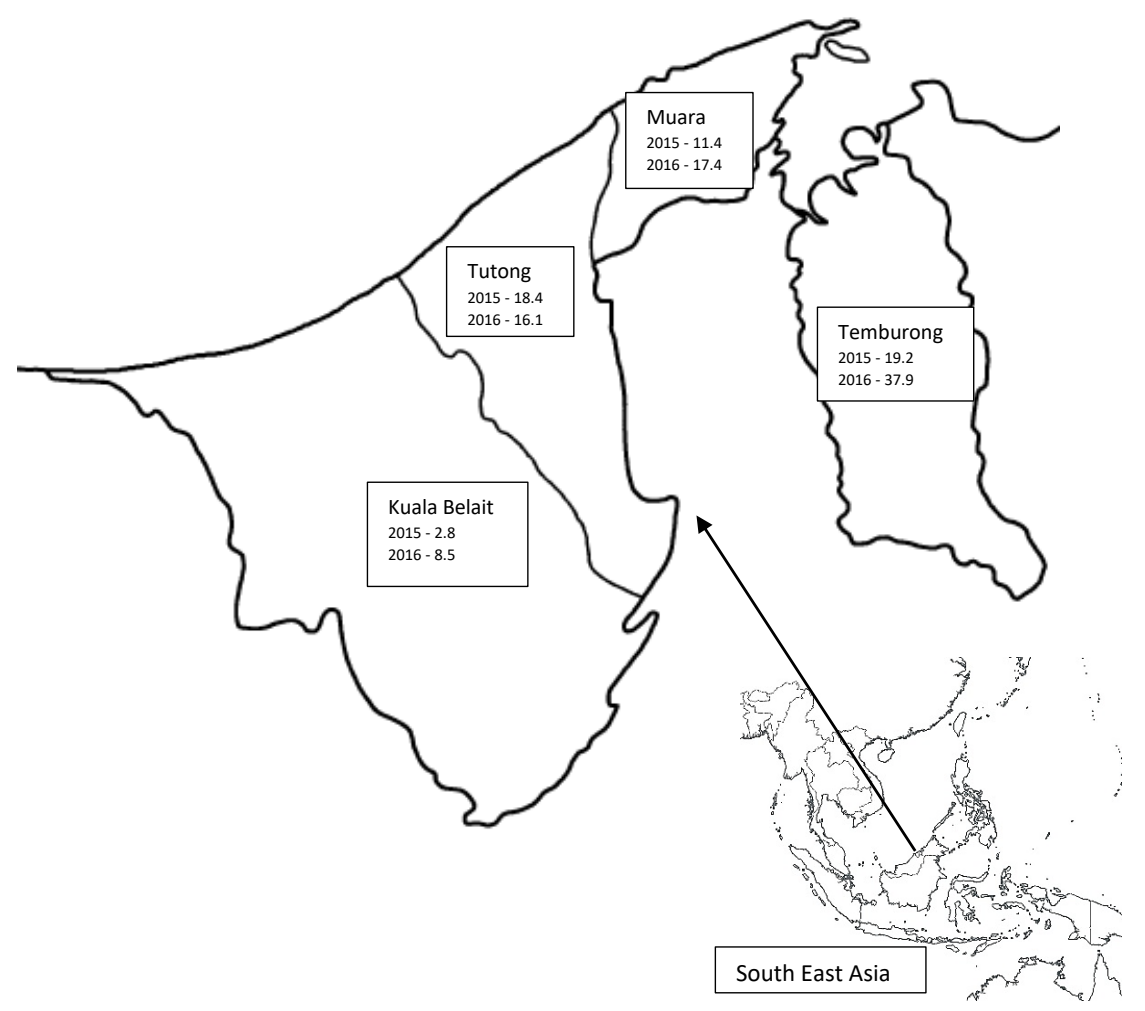

Figure 1. Map showing incidence/100,000 population for 2015 and 2016 by district, Brunei Darussalam.

\subsubsection{Month-Wise Number of New Cases}

The number of cases (2015 and 2016 combined) according to the month of admission is presented in Figure 2.

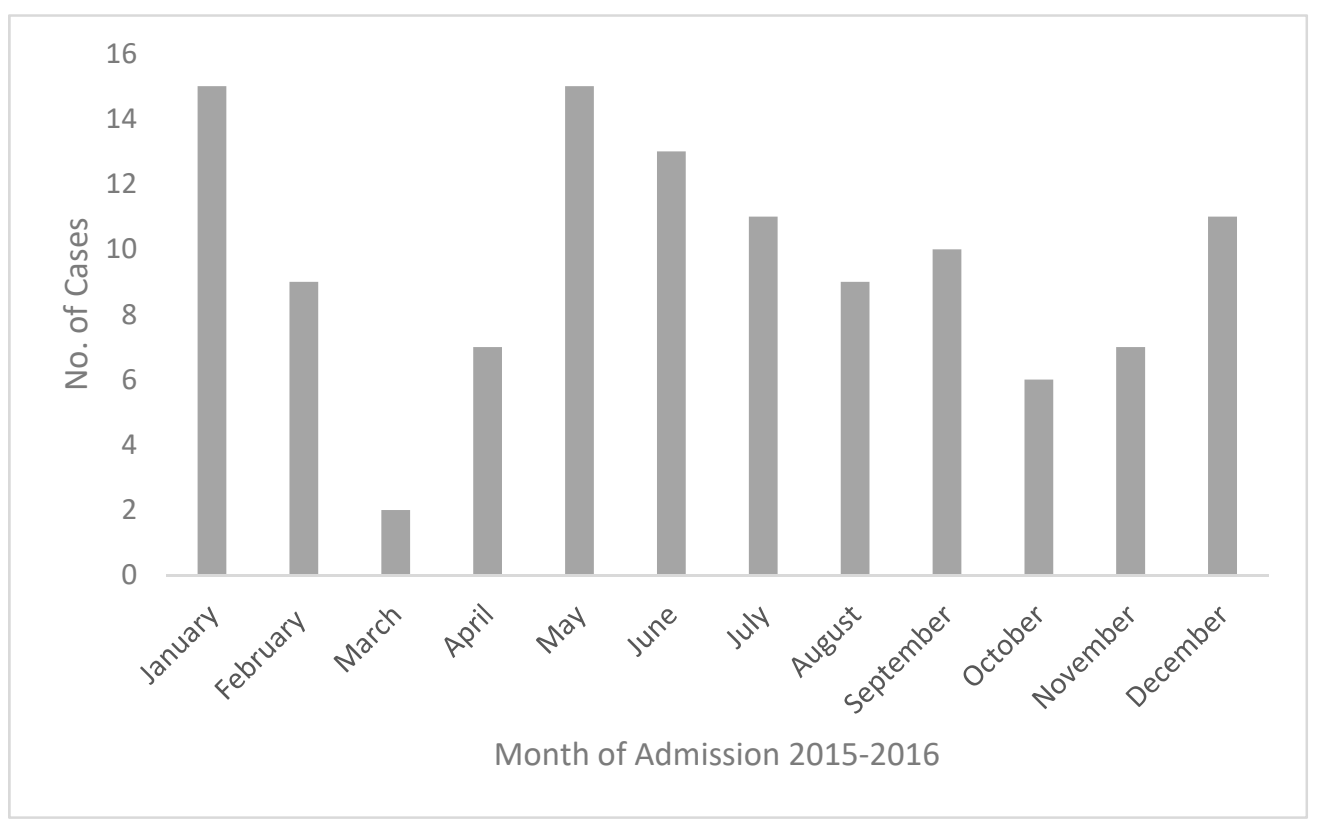

Figure 2. Meliodosis cases by month (2015-2016). 
The maximum number of admissions were recorded in the months of January and May with smaller peaks in June, July, and December.

\subsubsection{Risk Factors}

A number of risk factors were identified and are presented in Table 3.

Table 3. Risk Factors for melioidosis.

\begin{tabular}{ccc}
\hline Risk Factor & Cases & $\%$ \\
\hline Diabetes mellitus & 86 & 74.8 \\
Chronic renal disease & 23 & 20 \\
Cardiac disease & 17 & 14.8 \\
Lung diseases (COPD/TB) & 16 & 13.9 \\
Excessive alcohol intake & 6 & 5.2 \\
Thalassemia & 5 & 4.3 \\
Malignancy & 2 & 1.7 \\
None & 9 & 7.8
\end{tabular}

Note: Most of the chronic renal disease patients were those with end-stage renal failure on dialysis and thalassemia patients who were transfusion-dependent and had iron overload.

Diabetes mellitus was the most common risk factor, recorded in $74.8 \%$ of cases. More than one risk factor was present in $37.4 \%$ of cases, and there was no obvious risk factor seen in $7.8 \%(n=9)$.

\subsubsection{Clinical Presentations and Diagnosis}

Various clinical presentations were recorded (Table 4), pneumonia being the most common (47\%). In seven cases $(6 \%)$ no focus of infection could be found.

Table 4. Clinical presentation of melioidosis.

\begin{tabular}{ccc}
\hline Presentation & Cases & $\%$ \\
\hline Septic shock & 25 & 21.7 \\
Pneumonia & 54 & 47 \\
Soft tissue abscess & 21 & 18.3 \\
Musculo-skeletal & 10 & 8.7 \\
Neurological & 1 & 0.9 \\
No evidence of primary focus & 7 & 6 \\
\hline
\end{tabular}

Thirteen of 25 cases of septic shock had pneumonia as the primary source of infection, including in the 54 cases of pneumonia.

Internal organ abscesses were most commonly noted in spleen and liver (Table 5).

Table 5. Internal organ abscess in melioidosis.

\begin{tabular}{ccc}
\hline Organ & Cases & $\mathbf{\%}$ \\
\hline Spleen & 27 & 23.5 \\
Liver & 20 & 17.4 \\
Prostate & 5 & 6 \\
Lymph node & 4 & 3.5 \\
Kidney & 2 & 1.7 \\
Parotid & 1 & 0.9 \\
Brain & 1 & 0.9 \\
\hline
\end{tabular}

In most cases (67\%), the diagnosis was confirmed by blood culture (Table 6). 
Table 6. Source of culture for diagnosis of melioidosis.

\begin{tabular}{ccc}
\hline Modality & No. of Isolates & $\mathbf{\%}$ \\
\hline Blood culture & 77 & 67 \\
Fluid culture & 11 & 9.6 \\
Pus culture & 37 & 32.2 \\
\hline
\end{tabular}

CT-TAP was performed in 50.5\% $(n=58)$ of cases. The reasons for a patient not undergoing CT-TAP were septic shock and serious illness, presentation as cellulitis or soft tissue abscess, and issues with payment for expatriate workers.

\subsubsection{Outcome of Treatment}

In the years 2015 and 2016, there was an overall mortality of 27\% (Table 7). Mortality in patients presenting with septic shock was $64 \%$. The mortality in women was $41.9 \%(13 / 31)$ compared to $21.4 \%$ $(18 / 84)$ in men $(p<0.005)$.

Table 7. Outcome of treatment of cases.

\begin{tabular}{ccc}
\hline Outcome & Cases & $\%$ \\
\hline Recovery & 79 & 68.7 \\
Death & 31 & 27 \\
Relapse & 5 & 4.3 \\
\hline
\end{tabular}

Of the five cases with relapse, one patient died during readmission with septic shock (not included in the 31 fatalities).

In 2015,11 patients $(23.9 \%)$ were admitted with septic shock and the mortality was $26.1 \%(n=12)$ compared to 14 patients $(20 \%)$ and $27.5 \%(n=19)$, respectively, in 2016. Five of the nine patients without any risk factors succumbed to the disease.

\subsection{Discussion}

This is the first detailed epidemiological study of melioidosis from Brunei Darussalam. The data have revealed a slight increase in incidence from 11 to 16 per 100,000 population between 2015 and 2016. It also showed a high number of expatriate workers being affected. Diabetes mellitus and chronic renal disease were the most common risk factors, whilst pneumonia and soft tissue abscess were the most common clinical presentations. The data suggested a possible link with seasonal variation in rainfall with more cases diagnosed in the wet months. Interestingly the highest incidence rate was from the Temburong district, which has the highest annual rainfall. The mortality was $27 \%$, being higher in women and patients presenting with septic shock.

For the purpose of discussion, we have compared our results with other large epidemiological studies from Asia-Pacific published after 2010 [18-25]. Our findings of male preponderance, the median age of patients, diabetes mellitus and chronic renal disease as the most common risk factors, and pneumonia and soft tissue abscesses as the most common presentations, are similar to the studies reviewed.

Though occupational history was not consistently recorded in the hospital notes, similar to a previous study [8], 25 expatriate patients with varied nationalities (median age 41.5 years) were noted. Generally the majority of these were labourers working in the agriculture or construction industries, with a high chance of exposure to contaminated soil and water.

The overall incidences of 11 and 16.3 per 100,000 population in 2015 and 2016, respectively, are considerably higher than those reported from Thailand and Singapore [22,25]. It is important to note that, in Singapore, a recent decrease in incidence was reported [25], while the incidence has 
remained constant or increased in Australia and Thailand [18,22]. Our rates are comparable to the rate of 16.45/100,000 reported from the state of Kedah, Malaysia by Hassan et al. [21]. In contrast, another study based on the melioidosis registry from the Pahang state of Malaysia reported a much lower rate of 4.3/100,000 (adult 6.0/100,000 and paediatric 1.6/100,000) [26]. This reflects geographical differences, as seen in our study, with different rates between districts, even for a small country.

The current rates reported showed marked increase compared to the incidence rates (2.9 to 5.6/100,000 for 1993 and 1994, respectively) reported by Luqman et al. [5]. The upsurge may well be contributed to by better awareness and improvement in diagnostic facilities. Melioidosis was possibly under-diagnosed and under-reported in the past [27]. Another plausible reason for the upward trend is the rise in the overall population along with an increase in the number of people with risk factors, such as diabetes and chronic kidney disease.

There were four patients (3.5\%) under the age of 15 years, comparable to the rate reported by Hassan et al., (5.5\%), and higher than the rate reported by How et al., $(1.6 / 100,000)[21,26]$. Consistent with reports in the literature, our paediatric patients had no risk factors, presented with soft tissue abscesses, two of them during the wet months of December and January, and recovered completely with treatment [28]. Unlike Kingsley et al., we did not observe any cases of neonatal melioidosis [29].

In the present study, a higher number of cases was observed in the wet months of May to July and December to February, with $64.3 \%$ being admitted during these months. The incidence was also higher in the Temburong district, which records the highest rainfall amongst the four districts. A high level of moisture in the soil during months of high rainfall has been reported to correlate with an increased incidence of melioidosis [30,31]. This association has been reported from Australia [18,20], Singapore [25], Malaysia [21], and India [23]. In contrast, Limmathurotsakul et al. reported a negative association between the total annual rainfall and the number of cases in each year of their study from Thailand [22]. However, in this study monthly rainfall data and the number of cases per month were not available. Consistent with an incubation period of a few weeks, the incidence may also be higher in the corresponding period after high rainfall [32].

Diabetes mellitus was recorded as a risk factor in $74.8 \%$ of cases, similar to the proportion in India [23] and Malaysia [24]. Other studies have reported it in 39-57\% of their cases [18,20-22,25]. In two studies from Australia $[18,20]$, excessive alcohol use was the second most common risk factor. In the present study it was noted in $5.2 \%$ of cases but could be underreported due to a reluctance to volunteer such a history. However, the sale of alcohol is banned in Brunei Darussalam and, therefore, our finding is likely to be a true reflection of the real situation.

The number of patients with melioidosis without any risk factors was lower $(7.8 \%, n=9)$ in the present study compared to other studies (13-22\%) [18,20,23,24] but similar to that reported by Kingsley et al. [29]. It has been suggested that individuals with no risk factors have less severe symptoms and mortality is rare [29]. However, in the present study $5 / 9$ patients succumbed to the disease, three of whom presented with septic shock, and two with large soft tissue abscesses suggesting fulminant infection.

Consistent with the studies reviewed, pneumonia was the most common clinical presentation followed by soft tissue abscesses [18,20-25]. Septic shock was the mode of presentation in $21.7 \%(n=25)$ cases. In a review of case reports published from Malaysia, a high frequency of primary neurological presentation was noted (7.5\%) [29]. The rates reported by other authors $[18,21,23,24]$ and one case in the present study is much lower. This may be due to selection bias in the case reports reviewed.

Internal organ abscesses were most common in the spleen and liver, as noted in the literature $[18,21,23,24]$; however, the proportion was much higher in the present study. We had only one case of subdural empyema and no cases with mycotic aneurysms or pericardial involvement. The proportion of prostatic abscesses in the present study was $4.3 \%$; there is a wide variation reported in the literature, from $0.3 \%$ in Thailand [33], to a high of $20 \%$ in Australia [18]. Our finding of the frequency of parotid involvement $(n=1,0.9 \%)$ is comparable to other studies [23,24,29], but much lower than that reported in Thai children (20-30\%) [34]. We did not find any case with pancreatic 
involvement in the present study. The four cases previously reported were seen over a period of six years, compared to two years of the present study [7]. Moreover, only about $50 \%$ of patients in the present study underwent CT-TAP for various reasons, which could have resulted in under-reporting of internal organ abscesses.

The overall mortality in the present study was $27 \%$, with a higher mortality in women $(41.9 \%)$ compared to men $(21.4 \% ; p<0.05)$. This association has also been reported by Kingsley et al. [29]. Mortality was also significantly higher in patients who presented with septic shock.

There is a wide variation in the reported mortality in the literature, from $9.5 \%$ in India [23], $42.6 \%$ in Thailand [22], and between 32-63\% in Malaysia [19,21,24]. Decreasing mortality rates have been reported from Australia [18] and Singapore [25]. The reasons put forward for this are access to standardized health care and the institution of prompt treatment according to recommendations [35]. In Singapore, in particular, it is thought to be due to efforts to optimise diabetes care and enhanced environmental and water management [25]. Our current rate is slightly higher than that reported in the earlier study by Luqman et al., (20.3\%) [5], possibly due to late presentations and multiple comorbidities.

Five cases $(4.3 \%)$ had recurrence of the disease, which is a similar rate to that reported by Currie et al. [18]. One of the 31 cases that did not survive also presented with a recurrence. They had underlying risk factors of diabetes mellitus (4/6), malignancy (2/6), and chronic renal disease (1/6). Non-compliance with previous treatment was recorded in two cases. The institution of prompt treatment along international guidelines explains the lower current rate compared to relapses seen in $60 \%$ of cases in the past [5].

The strength of the present study is that, owing to the method of data collection, it can be taken as a true reflection of the disease state in the country. A limitation of the study was its retrospective nature. Data, particularly on occupation and alcohol abuse as risk factors, were not adequately recorded.

\section{Melioidosis in Animals}

All livestock farms in Brunei Darussalam are advised through the Department of Agriculture and Agrifood about good animal husbandry practices as a method of prevention of diseases.

According to the Animal Health and Disease Control Unit under the Livestock and Veterinary Services Division, 93 and 128 goats were treated for presumed melioidosis in 2015 and 2016, respectively. This was based on farmers' complaints and clinical findings, but no bacteriological evidence was obtained. A positive response to treatment was noted in $80 \%$ of cases. The high susceptibility of goats to melioidosis has been reported in the literature [36,37].

It has been suggested that goats and humans are exposed to similar levels of B. pseudomallei in the environment and, hence, the incidence is likely to be similar [37]. However, data on melioidosis incidence in animals are not available in Brunei Darussalam to confirm this.

In Brunei Darussalam, the goat farms are mostly small, with herds less than 500 head. The goat houses use raised flooring and practice 'cut and carry' grass for feeding to avoid direct contact of animals with the soil. Cattle livestock are imported from Australia, and Sabah and Sarawak in Malaysia, and kept in holding yards for seasonal sales and are then slaughtered shortly afterwards (short-term rearing), so that goats make up the longest-standing ruminant population. Buffaloes are less susceptible to melioidosis, as noted in a review [38].

In late 2016, a few goat and sheep carcasses were submitted for autopsy with lesions suspicious of melioidosis. The farm had recently carried out excavation work, followed by heavy rain. The animals were 2-6 months of age and, hence, thought to be immunocompromised. The bacteriological tests were negative for B. pseudomallei. The sick animals from the farm were treated and water was disinfected as a precautionary measure. Workers in the farm and fodder plantation with respiratory symptoms were advised to attend the nearest health centre. 


\section{Future Direction}

It is clear from the Health Information Booklet 2016, published by the Ministry of Health, Brunei Darussalam, that diabetes, pneumonia, and septicaemia are amongst the top 10 causes of death in the country [39]. The number of cases of melioidosis in $2016(n=69)$ was listed after the number of cases of gastroenteritis and food poisoning in the list of waterborne diseases. However, melioidosis does not feature in the list of notifiable diseases in Brunei Darussalam, unlike in Australia [20] and Singapore [25].

Evidence-based guidelines are now available for prevention of melioidosis in endemic areas [40]. Suntornsut et al. explored the barriers in implementing preventive measures for melioidosis and have suggested a number of interventions. Based on these, the authors have recommended a multifaceted intervention involving community and government agencies [41].

Kingsley et al. have stressed the importance of making melioidosis notifiable and starting registries in endemic countries to improve the efforts to effectively control the disease [29]. Through such registries, it will be possible to obtain reliable clinical and epidemiological information, note trends in the incidence, and determine effective treatment and mortality.

Involvement of the various stakeholders that encounter melioidosis in their practice and the formation of a national registry would help to ensure that data collected is complete and standardized. This will allow comparisons and the analysis of trends. Thus, the formation of a regional or international registry would allow the condition to be better studied. If Brunei Darussalam were to follow Australia and Singapore in making the condition a notifiable disease, this would further enhance awareness and this may have an impact on the clinical course of the disease in the future.

Acknowledgments: We are grateful to Diana Dennis and Raihan Zurhairah Bte Hj Zulkipli, Division of Livestock and Veterinary Services, Department of Agriculture and Agrifood, for their input on melioidosis in animals in Brunei Darussalam. We are also grateful to Woo Boon Chu, Clinical Microbiology Laboratory, Department of Laboratory Services, for help with retrieving a list of cases of melioidosis.

Author Contributions: K.P. conducted the data collection and analysis, and the writing and revision of the manuscript; K.A.A.K. conducted the data collection and revision of the manuscript; R.A. wrote and revised the manuscript; and V.H.C. wrote and revised the manuscript.

Conflicts of Interest: The authors declare no conflict of interest.

\section{References}

1. Limmathurotsakul, D.; Golding, N.; Dance, D.A.B.; Messina, J.P.; Pigott, D.M.; Moyes, C.L.; Rolim, D.B.; Bertherat, E.; Day, N.P.J.; Peacock, S.J.; et al. Predicted global distribution of Burkholderia pseudomallei and burden of melioidosis. Nat. Microbiol. 2016, 1. [CrossRef] [PubMed]

2. Geography of Brunei. Available online: https://en.wikipedia.org/wiki/Geography_of_Brunei (accessed on 3 January 2018).

3. Rainfall. Available online: https://www.bsp.com.bn/panagaclub/pnhs_old/geology/HTM/OVERVIEW/ CLIMATE/RAIN/rain1_1.HTM (accessed on 3 January 2018).

4. Climate. Available online: http://bruneiweather.com.bn/climate (accessed on 3 January 2018).

5. Luqman, N.; On, S.C.; Satyawany, N.; Da, P. Melioidosis in Brunei-epidemiological and clinical profile. Brunei Int. Med. J. 1999, 1, 291-297.

6. Lim, K.S.; Chong, V.H. Radiological manifestations of melioidosis. Clin. Radiol. 2010, 65, 66-72. [CrossRef] [PubMed]

7. Chong, V.H.; Lim, K.S.; Sharif, F. Pancreatic Involvement in melioidosis. JOP 2010, 11, 365-368. [PubMed]

8. Pande, K.C.; Hj Abdul Kadir, K.A. Melioidosis of the extremities in Brunei Darussalam. Singap. Med. J. 2011, 52, 346-350.

9. Khairul Azmi, A.K.; Sattayavani, M.; Pande, K. Melioidosis: Antibiogram of cases in Brunei Darussalam. Brunei Int. Med. J. 2014, 10, 19-24. 
10. Samad, I.; Wang, M.C.; Chong, V.H. Intracerebral coinfection with Burkholderia pseudomallei and Cryptococcus neoformans in a patient with systemic lupus erythematosus. Southeast Asian J. Trop. Med. Public Health 2014, 45, 352-356. [PubMed]

11. Chong, V.H.; Sharif, F.; Bickle, I. Urogenital melioidosis: A review of clinical presentations, characteristic and outcomes. Med. J. Malays. 2014, 69, 257-260.

12. Peacock, S.J.; Chieng, G.; Cheng, A.C.; Dance, D.A.B.; Amornchai, P.; Wongsuvan, G.; Teerawattanasook, N.; Chierakul, W.; Day, N.P.J.; Wuthiekanun, V. Comparison of Ashdown's medium, Burkholderia cepacia medium, and Burkholderia pseudomallei selective agar for clinic isolation of Burkholderia pseudomallei. J. Clin. Microbiol. 2005, 43, 5359-5361. [CrossRef] [PubMed]

13. Podin, Y.; Kaestil, M.; McMahon, N.; Hennessy, J.; Ngian, H.U.; Wong, J.S.; Mohana, A.; Wong, S.C.; William, T.; Mayo, M.; et al. Reliability of automated biochemical identification of Burkholderia pseudomallei is regionally dependent. J. Clin. Microbiol. 2013, 51, 3076-3078. [CrossRef] [PubMed]

14. Chetchotisakd, P.; Chierakul, W.; Chaowagul, W.; Anunnatsiri, S.; Phimda, K.; Mootsikapun, P.; Chaisuksant, S.; Pilaikul, J.; Thinkhamrop, B.; Phiphitaporn, S.; et al. Trimethoprim-sulfamethoxazole versus trimethoprim-sulfamethoxazole plus doxycycline as oral eradicative treatment for melioidosis (MERTH): A multicentre, double-blind, non-inferiority, randomised controlled trial. Lancet 2014, 383, 807-814. [CrossRef]

15. Dance, D. Treatment and prophylaxis of melioidosis. Int. J. Antimicrob. Agents 2014, 43, 310-318. [CrossRef] [PubMed]

16. Centers for Diseases Control and Prevention. Biosafety in microbiological and biomedical laboratories. Section IV. Laboratory biosafety level criteria. Available online: https://www.cdc.gov/biosafety/ publications/bmbl5/bmbl5_sect_iv.pdf (accessed on 9 February 2018).

17. Population. Available online: http://www.depd.gov.bn/sitePages/Population.aspx (accessed on 3 January 2018).

18. Currie, B.J.; Ward, L.; Cheng, A.C. The epidemiology and clinical spectrum of Melioidosis: 540 cases from the 20 year Darwin prospective study. PLoS Negl. Trop. Dis. 2010, 4, e900. [CrossRef] [PubMed]

19. Deris, Z.Z.; Hasan, H.; Siti Suraiya, M.N. Clinical characteristics and outcomes of bacteraemic melioidosis in a teaching hospital in a northeastern state of Malaysia. J. Infect. Dev. Ctries. 2010, 4, 430-435. [PubMed]

20. Hanna, J.N.; Humphreys, J.L.; Brookes, D.L.; Messina, T.; Raulli, A. Melioidosis in North Queensland, 2000-2009. Commun. Dis. Intell. 2010, 34, 444-447.

21. Hassan, M.R.A.; Pani, S.P.; Peng, N.P.; Voralu, K.; Vijayalakshmi, N.; Mehandrakar, R.; Aziz, N.A.; Michael, E. Incidence, risk factors and clinical epidemiology of melioidosis: A complex socio-ecological emerging infectious disease in the Alor Setar region of Kedah, Malaysia. BMC Infect. Dis. 2010, 10, 302. [CrossRef] [PubMed]

22. Limmathurotsakul, D.; Wongratanacheewin, S.; Teerawattanasook, N.; Wongsuvan, G.; Chaisuksant, S.; Chetchotisakd, P.; Chaowagul, W.; Day, N.P.J.; Peacock, S.J. Increasing incidence of human melioidosis in northeast Thailand. Am. J. Trop. Med. Hyg. 2010, 82, 1113-1117. [CrossRef] [PubMed]

23. Vidyalakshmi, K.; Lipika, S.; Vishal, S.; Damodar, S.; Chakrapani, M. Emerging clinico-epidemiological trends in melioidosis: Analysis of 95 cases from western coastal India. Int. J. Infect. Dis. 2012, 16, e491-e497. [CrossRef] [PubMed]

24. Zueter, A.; Yean, C.Y.; Abumarzouq, M.; Rahman, Z.A.; Deris, Z.Z.; Harun, A. The epidemiology and clinical spectrum of melioidosis in a teaching hospital in North-Eastern state of Malaysia: A fifteen-year review. BMC Infect. Dis. 2016, 16, 333. [CrossRef] [PubMed]

25. Pang, L.; Harris, P.N.A.; Seiler, R.L.; Ooi, P.L.; Cutter, J.; Goh, K.T.; Cook, A.R.; Fisher, D.; Chai, L.Y.A. Melioidosis, Singapore, 2003-2014. Emerg. Infect. Dis. 2018, 24, 140-143. [CrossRef] [PubMed]

26. How, S.H.; Ng, T.H.; Jamalludin, A.R.; Tee, H.P.; Kuan, Y.C.; Alex, F.; Sc, M.; Aminudin, C.A.; Sapari, S.; Quazi, M.H. Pahang melioidosis registry. Med. J. Malays. 2009, 64, 27-30.

27. Foong, Y.C.; Tan, M.; Bradbury, R.S. Melioidosis: A review. Rural Remote Health 2014, 14, 2763. [PubMed]

28. McLeod, C.; Morris, P.S.; Bauert, P.A.; Kilburn, C.J.; Ward, L.M.; Baird, R.W.; Currie, B.J. Clinical presentation and medical management of melioidosis in children: A 24-year prospective study in the Northern Territory of Australia and review of the literature. Clin. Infect. Dis. 2015, 60, 21-26. [CrossRef] [PubMed]

29. Kingsley, P.V.; Leader, M.; Nagodawithana, N.S.; Tipre, M.; Sathaikumar, N. Melioidosis in Malaysia: A review of case reports. PLoS Negl. Trop. Dis. 2016, 10, e0005182. [CrossRef] [PubMed] 
30. Liu, X.; Pang, L.; Sim, S.H.; Goh, K.T.; Ravikumar, S.; Win, M.S.; Tan, G.; Cook, A.R.; Fisher, D.; Chai, L.Y. Association of melioidosis incidence with rainfall and humidity, Singapore, 2003-2012. Emerg. Infect. Dis. 2015, 21, 159-162. [CrossRef] [PubMed]

31. Merritt, A.J.; Inglis, T.J.J. The role of climate in the epidemiology of melioidosis. Curr. Trop. Med. Rep. 2017, 4, 185-191. [CrossRef] [PubMed]

32. Currie, B.J.; Fischer, D.A.; Anstey, N.M.; Jacups, S.P. Melioidosis: Acute and chronic disease, relapse and re-activation. Trans. R. Soc. Trop. Med. Hyg. 2000, 94, 301-304. [CrossRef]

33. Suputtamongkol, Y.; Hall, A.J.; Dance, D.A.; Chaowagul, W.; Rajchanuvong, A.; Smith, M.D.; White, N.J. The epidemiology of melioidosis in Ubon Ratchatani, northeast Thailand. Int. J. Epidemiol. 1994, 23, 1082-1090. [CrossRef] [PubMed]

34. Cheng, A.C.; Currie, B.J. Melioidosis: Epidemiology, pathophysiology and management. Clin. Microbiol. Rev. 2005, 18, 383-416. [CrossRef] [PubMed]

35. Wiersinga, W.J.; Currie, B.J.; Peacock, S.J. Melioidosis. N. Engl. J. Med. 2012, 367, 1035-1044. [CrossRef] [PubMed]

36. Choy, J.L.; Mayo, M.; Janmaat, A.; Currie, B.J. Animal melioidosis in Australia. Acta Trop. 2000, 74, $153-158$. [CrossRef]

37. Limmathurotsakul, D.; Thammasart, S.; Warrasuth, N.; Thapanagulsak, P.; Jatapai, A.; Pengreungrojanachai, V.; Anun, S.; Joraka, W.; Thongkam koon, P.; Saiyen, P.; et al. Melioidosis in animals, Thailand, 2006-2010. Emerg. Infect. Dis. 2012, 18, 325-327. [CrossRef] [PubMed]

38. Sprague, L.D.; Neubauer, H. Melioidosis in animals: A review of epizootiology, diagnosis and clinical presentation. J. Vet. Med. 2004, 51, 305-320. [CrossRef] [PubMed]

39. Health Information Booklet 2016. Available online: www.moh.gov.bn/.../Health\%20Indicator\%20Booklet/ HIB_2016.pdf (accessed on 3 January 2018).

40. Limmathurotsakul, D.; Kanoksil, M.; Wuthiekanun, V.; Kitphati, R.; de Stavola, B.; Day, N.P.J.; Peacock, S.J. Activities of daily living associated with acquisition of melioidosis in northeast Thailand: A matched case-control study. PLoS Negl. Trop. Dis. 2013, 7, e2072. [CrossRef] [PubMed]

41. Suntornsut, P.; Wongsuwan, N.; Malasit, M.; Kitphati, R.; Michie, S.; Peacock, S.J.; Limmathruotsakul, D. Barriers and recommended interventions to prevent melioidosis in northeast Thailand: A focus group study using the behaviour change wheel. PLoS Negl. Trop. Dis. 2016, 10, e0004823. [CrossRef] [PubMed] 